International Journal of Pure and Applied Mathematics

Volume 84 No. 5 2013, 583-591

ISSN: 1311-8080 (printed version); ISSN: 1314-3395 (on-line version)

url: http://www.ijpam.eu

doi: http://dx.doi.org/10.12732/ijpam.v84i5.11

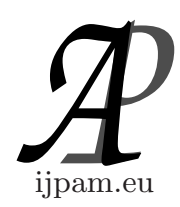

\title{
NONLINEAR EIGENVALUE PROBLEM WITHOUT AMBROSETTI AND RABINOWITZ CONDITION: AN ORLICZ SPACE SETTING
}

\author{
Nawel Benouhiba ${ }^{1}$, Hacene Saker ${ }^{2}$ \\ ${ }^{1,2}$ Department of Mathematics \\ LMA, Badji Mokhtar-Annaba University \\ P.O. Box 12 El Hadjar Annaba 23000, ALGERIA
}

Abstract: We study the Dirichlet boundary value problem for the $p(x)$ Laplacian of the form

$$
\begin{cases}-\Delta_{p(x)} u=\lambda f(x, u), & x \in \Omega \\ u=0 & x \in \partial \Omega .\end{cases}
$$

We introduce a new variational technic that allows us to investigate problem (P) without need of the Ambrosetti and Rabinowitz condition on the nonlinearity $f$.

AMS Subject Classification: 35B45, 35J60, 35J70

Key Words: $p(x)$-Laplacian, Variable exponent Lebesgue-Sobolev spaces, eigenvalue, critical point

\section{Introduction}

The purpose of this paper is to study the existence of nontrivial solutions for the following nonlinear boundary value problem involving the $p(x)$-Laplacian

$$
\begin{cases}-\Delta_{p(x)} u=\lambda f(x, u), & x \in \Omega \\ u=0 & x \in \partial \Omega\end{cases}
$$

Received: December 6, 2012

(c) 2013 Academic Publications, Ltd.

${ }^{\S}$ Correspondence author url: www.acadpubl.eu 
where $\Omega \subset \mathbb{R}^{N}, N>1$ is a bounded domain with smooth boundary, $\Delta_{p(x)} u=$ $\operatorname{div}\left(|\nabla u|^{p(x)-2} \nabla u\right)$ is the $p(x)$-Laplacian, $p \in C(\bar{\Omega})$ and the nonlinearity $f$ : $\mathbb{R}^{N} \times \mathbb{R} \longrightarrow \mathbb{R}$ is assumed to be a carathéodory function.

The considered eigenvalue problem involves variable exponent growth conditions. The study of such kind of equations is a very active field of investigation in the last decade since they can serve as a models for different physical phenomena. We refer to [8] for a model of partial differential equation with non standard growth in Electrorheological fluids.

The main idea of this work is to study equation (1) without assuming the Ambrosetti and Rabinowitz condition on $f$, namely, there exist $\theta>2$ and $t_{0}>0$ such that

$$
0<\theta F(x, t) \leq t f(x, t),|t| \geq t_{0}, \forall x \in \Omega
$$

where $F(x, t)=\int_{0}^{t} f(x, s) d s$, since it is a restrictive condition eliminating many nonlinearities.

In [7], the equation (1) has been considered in the case of constant exponent. While in [3], Fan, Zhang and Zhao showed that the problem

$$
-\Delta_{p(x)} u=\lambda|u|^{p(x)-2} u
$$

has infinitely many positive eigenvalues.

M. Mihăilescu and V. Rădulescu in [5] have studied the problem

$$
-\Delta_{p(x)} u=\lambda|u|^{q(x)-2} u
$$

where $p(x) \neq q(x)$ and proved the existence of a continuous family of eigenvalues which lies in a neighborhood of the origin.

The familiar approach to solve problem (1) is to search critical points of the functional

$$
L(u)=\int_{\Omega} \frac{1}{p(x)}|\nabla u|^{p(x)} d x-\lambda \int_{\Omega} F(x, u) d x
$$

In this method, one must assume in general that the nonlinearity $f$ satisfies the (AR) condition. The author in [7] discovered a new functional whose critical point is a solution of problem (1) in the special case $p(x)=p=$ constant.

In this paper, we use a more general functional and we get the existence of infinitely many solutions of problem(1) without assuming the (AR) condition.

We study problem (1.1) under the following assumptions:

$$
p, q \in C(\bar{\Omega}) \text { and } 1<p(x)<q(x)<p^{*}(x)<\infty, \forall x \in \bar{\Omega} .
$$

The function $f: \Omega \times\left[0, \infty\left[\longrightarrow \mathbb{R}^{+}\right.\right.$satisfies the Carathéodory condition, namely, $f(., t)$ is measurable for all $t \in[0, \infty[$ and $f(x,$.$) is continuous for almost all$ $x \in \Omega$. 
There exists an open no empty $\omega \subset \Omega$, and $0 \leq \tau_{1} \leq \tau_{2} \leq \infty$ such that

$$
f(x, t)>0 \text { on } \omega \times] \tau_{1}, \tau_{2}[
$$

and there exists $g \in L^{s(x)}(\Omega) \cap L^{\infty}(\Omega)$ such that

$$
f(x, t) \leq g(x) t^{q(x)-1}
$$

for all $x \in \Omega$ and $t \geq 0$ where the variable exponent $s \in C(\bar{\Omega})$ satisfies $\frac{p^{*}(x)}{p^{*}(x)-q(x)}<s(x)$ for all $x \in \Omega$.

In what follows, the Letter $c$ will be indiscriminately used to denote various constants when the exact values are irrelevant.

\section{Generalized Lebesgue and Sobolev Spaces}

We recall in what follows some basic facts about the variable exponent Lebesgue and Sobolev spaces $L^{p(x)}(\Omega)$ and $W_{0}^{1, p(x)}(\Omega)$.

Set

$$
C_{+}(\bar{\Omega})=\{p ; p \in C(\bar{\Omega}), \quad p(x)>1 \text { for all } x \in \bar{\Omega}\}
$$

We define

$$
p^{-}=\underset{x \in \Omega}{\operatorname{essinf}} p(x), \quad p^{+}=\underset{x \in \Omega}{\operatorname{ess} \sup } p(x) .
$$

For any $p \in C_{+}(\bar{\Omega})$ we define the variable exponent Lebesgue space $L^{p(x)}(\Omega)$ by

$$
L^{p(x)}(\Omega)=\left\{f ; f: \Omega \rightarrow \mathbb{R}, \int_{\Omega}|f(x)|^{p(x)} d x<\infty\right\}
$$

with the norm

$$
|f|_{p(x)}=\inf \left\{\lambda>0: \int_{\Omega}\left|\frac{f(x)}{\lambda}\right|^{p(x)} d x \leq 1\right\} .
$$

Variable exponent Lebesgue space $L^{p(x)}(\Omega)$ is a Banach space. It is reflexive if and only if $1<p^{-} \leq p^{+}<\infty$ (see [4]).

The inclusion between Lebesgue spaces generalizes naturally: if $0<|\Omega|<$ $\infty$ and $r_{1}, r_{2}$ are variable exponents such that $r_{1}(x) \leq r_{2}(x)$ almost everywhere in $\Omega$ then there exists a continuous embedding $L^{r_{2}}(\Omega) \hookrightarrow L^{r_{1}}(\Omega)$.

Let $L^{p^{\prime}(x)}(\Omega)$ denote the conjugate space of $L^{p(x)}(\Omega), \frac{1}{p(x)}+\frac{1}{p^{\prime}(x)}=1$. For any $u \in L^{p(x)}(\Omega)$ and $v \in L^{p^{\prime}(x)}(\Omega)$ the Hölder type inequality 


$$
\left|\int_{\Omega} u v d x\right| \leq\left(\frac{1}{p^{-}}+\frac{1}{p^{\prime-}}\right)|u|_{p(x)}|v|_{p^{\prime}(x)}
$$

holds true [4].

We recall that the modular of the $L^{p(x)}(\Omega)$ is the mapping $\rho_{p(x)}: L^{p(x)}(\Omega) \rightarrow$ $\mathbb{R}$ defined by

$$
\rho_{p(x)}(u)=\int_{\Omega}|u|^{p(x)} d x .
$$

In the case $p^{+}<\infty$ then for $f_{n}, f \in L^{p(x)}(\Omega)$ we have

$$
\begin{gathered}
|f|_{p(x)}^{p^{-}} \leq \varrho_{p(x)}(f) \leq|f|_{p(x)}^{p^{+}} \quad \text { if } \quad|f|_{p(x)}>1, \\
|f|_{p(x)}^{p^{+}} \leq \varrho_{p(x)}(f) \leq|f|_{p(x)}^{p^{-}} \quad \text { if } \quad|f|_{p(x)} \leq 1 . \\
f_{n} \rightarrow f \text { in } L^{p(x)}(\Omega) \text { if and only if } \varrho_{p(x)}\left(f_{n}-f\right) \rightarrow 0 .
\end{gathered}
$$

Proposition 2.1. [2] Let $q \in L^{\infty}(\Omega)$ be such that $1 \leq p(x) q(x) \leq \infty$ for a. e. in $\Omega$. Let $f \in L^{q(x)}(\Omega), f \neq 0$. Then

$$
\begin{aligned}
& |f|_{p(x) q(x)}^{q^{-}} \leq\left.\left.|| f\right|^{q(x)}\right|_{p(x)} \leq|f|_{p(x) q(x)}^{q^{+}} \quad \text { if }|f|_{p(x) q(x)}>1 \\
& |f|_{p(x) q(x)}^{q^{+}} \leq\left.\left.|| f\right|^{q(x)}\right|_{p(x)} \leq|f|_{p(x) q(x)}^{q^{-}} \quad \text { if }|f|_{p(x) q(x)} \leq 1
\end{aligned}
$$

In particular, if $q(x)=q$ is constant then

$$
\left.\left.|| f\right|^{q}\right|_{p(x)}=|f|_{p(x)}^{q}
$$

We define $W_{0}^{1, p(x)}(\Omega)$ as the closure of $C_{0}^{\infty}(\Omega)$ under the norm

$$
\|f\|_{1, p(x)}=|\nabla f|_{p(x)}
$$

The space $W_{0}^{1, p(x)}(\Omega)$ a separable and reflexive Banach space. We note that if $q \in C_{+}(\bar{\Omega})$ and $q(x)<p^{*}(x)$ for all $x \in \bar{\Omega}$ then the embedding $W_{0}^{1, p(x)}(\Omega) \hookrightarrow$ $L^{q(x)}(\Omega)$ is compact and continuous, where $p^{*}(x)=\frac{N p(x)}{N-p(x)}$ if $p(x)<N$ or $p^{*}(x)=+\infty$ if $p(x) \geq N$. We refer to $[2,4,6]$ for further properties of variable exponent Lebesgue and Sobolev spaces. 


\section{Problem Position and Existing Results}

We say that $\lambda \in \mathbb{R}$ is an eigenvalue of Problem (1.1) if there exists $u \in$ $W_{0}^{1, p(x)}(\Omega), u \not \equiv 0$ such that

$$
\int_{\Omega}|\nabla u|^{p(x)-2} \nabla u \cdot \nabla v d x=\lambda \int_{\Omega} f(x, u) v d x
$$

for any $v \in W_{0}^{1, p(x)}(\Omega)$. Such solution is called weak solution.

We denote by $E$ the set of nondecreasing $C^{1}\left(\mathbb{R}^{+}, \mathbb{R}^{+}\right)$functions $\phi$ that satisfy: there exists $c>0$ such that

$$
\phi(r) \geq\left\{\begin{array}{c}
c r^{\frac{s^{-}}{p^{+}}} \text {for } 0<r<1 \\
c r^{\frac{s^{+}}{p^{-}}} \text {for } r \geq 1 .
\end{array}\right.
$$

It is clear that $E \neq \emptyset$. We define on $E$ an equivalence relation $\sim$ by

$$
\forall \phi_{1}, \phi_{2} \in E: \phi_{1} \sim \phi_{2} \Leftrightarrow \exists c>0: \phi_{1}(r)=c \phi_{2}(r), \forall r>0 .
$$

We denote by $\Phi$ the elements of $\bar{E}=E_{/ \sim}$.

For any $\Phi \in \bar{E}$ we define

$$
J_{\Phi}: W_{0}^{1, p(x)}(\Omega)-\{0\} \rightarrow \mathbb{R}^{+}
$$

by

$$
J_{\Phi}(u)=\frac{\int_{\Omega} F(x, u) d x}{\Phi(I(u))}
$$

where

$$
I(u)=\int_{\Omega} \frac{1}{p(x)}|\nabla u|^{p(x)} d x
$$

and

$$
F(x, t)=\int_{o}^{t} f(x, s) d s .
$$

Lemma 3.1. For all $\Phi \in \bar{E}, J_{\Phi}$ is well defined and bounded from above in $W_{0}^{1, p(x)}(\Omega)-\{0\}$.

Proof. It follows from the assumptions that $J_{\Phi}$ is well defined. Applying the Hölder inequality we get for any $u \in W_{0}^{1, p(x)}(\Omega)$

$$
\int_{\Omega} F(x, u) d x \leq\left.\left.\frac{c_{s}}{q^{-}}|g|_{s(x)}|| u\right|^{q(x)}\right|_{s^{\prime}(x)} .
$$


So

$$
\int_{\Omega} F(x, u) d x \leq \frac{c_{s}}{q^{-}}|g|_{s(x)}|u|_{q(x) s^{\prime}(x)}^{s^{i}}, i=+o r-.
$$

By assumption $\frac{p^{*}(x)}{p^{*}(x)-q(x)}<s(x)$ so $q(x) s^{\prime}(x)<p^{*}(x)$. It follows that $W_{0}^{1, p(x)}(\Omega)$ is continuously injected in $L^{q(x) s^{\prime}(x)}(\Omega)$. So, there exists $c>0$ such that

$$
\int_{\Omega} F(x, u) d x \leq c\|u\|_{1, p(x)}^{s^{i}} \leq c\left(\|u\|_{1, p(x)}^{s^{+}}+\|u\|_{1, p(x)}^{s^{-}}\right) .
$$

If $\|u\|_{1, p(x)} \leq 1$ then $I(u) \leq 1$ and we get from 12

$$
\begin{gathered}
0 \leq J_{\Phi}(u) \leq c \frac{\left(\|u\|_{1, p(x)}^{s^{+}}+\|u\|_{1, p(x)}^{s^{-}}\right)}{\Phi(I(u))} \leq c \frac{\left(\|u\|_{1, p(x)}^{s^{+}}+\|u\|_{1, p(x)}^{s^{-}}\right)}{\frac{1}{2}(I(u))^{\frac{s^{-}}{p^{+}}}+\frac{1}{2}(I(u))^{\frac{s^{-}}{p^{+}}}} \\
\leq c \frac{\left(\|u\|_{1, p(x)}^{s^{+}}+\|u\|_{1, p(x)}^{s^{-}}\right)}{\frac{1}{2}(I(u))^{\frac{s^{-}}{p^{+}}}+\frac{1}{2}(I(u))^{\frac{s^{+}}{p^{+}}}}
\end{gathered}
$$

We have by $(2.3)$

$$
\|u\|_{1, p(x)} \leq\left(\varrho_{p(x)}(\nabla u)\right)^{\frac{1}{p^{+}}} \leq\left(\frac{1}{p^{+}} I(u)\right) \frac{1}{p^{+}} \leq(I(u))^{\frac{1}{p^{+}}} .
$$

It yields from 14 that

$$
0 \leq J_{\Phi}(u) \leq c
$$

We use the same argument for the case $\|u\|_{1, p(x)}>1$ to get the boundedness of $J_{\Phi}$.

Proposition 3.2. There exists $u_{\Phi} \in W_{0}^{1, p(x)}(\Omega)-\{0\}$ such that

$$
M=J_{\Phi}\left(u_{\Phi}\right)=\max _{u \in W_{0}^{1, p(x)}(\Omega)-\{0\}} J_{\Phi}(u) .
$$

Proof. From Lemma 3.2 it follows that $0 \leq M \in \mathbb{R}$. Let $\psi \in C_{0}^{\infty}(\Omega)$ such that $\operatorname{Supp} \psi \subset \omega$ and $\sup _{x \in \Omega} \psi(x)>\tau_{0}$. So, it yields $F(x, \psi)>0$. Thus, $M_{0}=J_{\Phi}(\psi)>0$.

Let $\left(u_{n}\right) \subset W_{0}^{1, p(x)}(\Omega)-\{0\}$ be an maximizing sequence. We may assume that $M \geq J_{\Phi}\left(u_{n}\right) \geq M_{0}$. We suppose that $u_{n}$ is not bounded in $W_{0}^{1, p(x)}(\Omega)$. So we can assume that $\left\|u_{n}\right\|_{1, p(x)} \geq 1$. Let $\varepsilon>\frac{s^{-}}{p^{-}}$and $\gamma$ such that $s^{+}<\gamma<$ $s^{-}+\varepsilon \frac{p^{-}}{s^{-}}\left(s^{+}-s^{-}\right)$be fixed.

$$
\left\|u_{n}\right\|_{1, p(x)}^{\gamma} \leq\left(\varrho_{p(x)}\left(\nabla u_{n}\right)\right)^{\frac{\gamma}{p^{-}}} \leq c\left(I\left(u_{n}\right)\right)^{\frac{\gamma}{p^{-}}}=c\left(I\left(u_{n}\right)\right)^{\frac{\gamma}{p^{-}}-\varepsilon}\left(I\left(u_{n}\right)\right)^{\varepsilon} .
$$


Applying the Young inequality, we get

$$
\left\|u_{n}\right\|_{1, p(x)}^{\gamma} \leq c^{\prime}\left(I\left(u_{n}\right)\right)^{\left(\frac{\gamma}{p^{-}}-\varepsilon\right) \beta}+c^{\prime \prime}\left(I\left(u_{n}\right)\right)^{\varepsilon \beta^{\prime}}
$$

for some $\beta$ that satisfies $\frac{s-}{\gamma-\varepsilon p^{-}}<\beta<\frac{s^{+}}{s^{+}-\varepsilon p^{-}}$. This choice ensures that $\varepsilon \beta^{\prime}<\frac{s^{+}}{p^{-}}$ and $\left.\frac{\gamma}{p^{-}}-\varepsilon\right) \beta<\frac{s^{-}}{p^{-}}$.

Hence

$$
\begin{gathered}
\left\|u_{n}\right\|_{1, p(x)}^{\gamma} \leq c^{\prime}\left(I\left(u_{n}\right)\right)^{\frac{s^{+}}{p^{-}}}+c "\left(I\left(u_{n}\right)\right)^{\frac{s^{-}}{p^{-}}} \leq c\left(I\left(u_{n}\right)\right)^{\frac{s^{+}}{p^{-}}} \\
\leq c \Phi\left(I\left(u_{n}\right)\right) \leq \frac{c}{M_{0}} \int_{\Omega} F\left(x, u_{n}\right) d x \leq C\left(\left\|u_{n}\right\|_{1, p(x)}^{s^{+}}+\left\|u_{n}\right\|_{1, p(x)}^{s^{-}}\right) \leq c\left\|u_{n}\right\|_{1, p(x)}^{s^{+}} .
\end{gathered}
$$

The constant $\gamma$ is greater than $s^{+}$. It follows that $u_{n}$ is bounded. So we can find a subsequence still denoted by $u_{n}$ that converges weakly to some $u_{\Phi} \in$ $W_{0}^{1, p(x)}(\Omega)$. By the Sobolev embedding, $u_{n}$ converges strongly in $L^{r(x)}(\Omega)$ for all $r$ that satisfies $p(x)<r(x)<p^{*}(x)$ for all $x \in \Omega$.

Let $\alpha(x)$ be such that $p(x)<\alpha(x) q(x)<p^{*}(x)$. It is knowing that under assumption (1.4), the Nemytskii operator $F(x,$.$) is continuous from L^{\alpha(x) q(x)}(\Omega)$ into $L^{\alpha(x)}$ and since $u_{n}$ is strongly convergent in $L^{\alpha(x) q(x)}(\Omega)$, then $F\left(., u_{n}\right)$ is strongly convergent in $L^{\alpha(x)}(\Omega)$. It follows that

$$
\int_{\Omega} F\left(x, u_{n}\right) d x \rightarrow \int_{\Omega} F\left(x, u_{\Phi}\right) d x .
$$

Since $\Phi$ is a nondecreasing continuous function and $I$ is w.l.s.c. (see [1]) we have that

$$
\Phi\left(I\left(u_{\Phi}\right)\right) \leq \liminf _{n \rightarrow \infty} \Phi\left(I\left(u_{n}\right)\right)
$$

And consequently

$$
M \geq J_{\Phi}\left(u_{\Phi}\right) \geq \limsup _{n \rightarrow \infty} J_{\Phi}\left(u_{n}\right)=M .
$$

We conclude that $J_{\Phi}$ attends its maximum at $u_{\Phi}$. We have $J_{\Phi}\left(u_{\Phi}\right)>0$. It follows that $\int_{\Omega} F\left(x, u_{\Phi}\right) d x>0$ and then $u_{\Phi} \neq 0$ since $F(x, 0)=0$.

We give now the following theorem.

Theorem 3.3. Suppose that the assumptions (1.2), (1.3) and (1.4) are fulfilled. Then, Problem (1.1) has infinitely many positive eigenvalues. 
Proof of Theorem 3.3. Standard arguments show that $J_{\Phi} \in C^{1}\left(W_{0}^{1, p(x)}(\Omega), \mathbb{R}\right)$ for all $\Phi \in \bar{E}$. By Proposition 3.2, it follows that $J_{\Phi}$ attends its maximum at $u_{\Phi} \in W_{0}^{1}, p(x)(\Omega)-\{0\}$. It yields that

$$
\left\langle J_{\Phi}^{\prime}\left(u_{\Phi}\right), v\right\rangle=0 \text { for all } v \in W_{0}^{1, p(x)}(\Omega) .
$$

By simple calculation we get

$$
\begin{aligned}
& \Phi^{\prime}\left(I\left(u_{\Phi}\right)\right)\left\langle I^{\prime}\left(u_{\Phi}\right), v\right\rangle \int_{\Omega} F\left(x, u_{\Phi}\right) d x \\
& \quad=\Phi\left(I\left(u_{\Phi}\right)\right) \int_{\Omega} f\left(x, u_{\Phi}\right) v d x \text { for all } v \in W_{0}^{1, p(x)}(\Omega)
\end{aligned}
$$

where

$$
\left\langle I^{\prime}(u), v\right\rangle=\int_{\Omega}|\nabla u|^{p(x)-2} \nabla u \cdot \nabla v d x .
$$

This is equivalent to

$$
\int_{\Omega}\left|\nabla u_{\Phi}\right|^{p(x)-2} \nabla u_{\Phi} \cdot \nabla v d x=\lambda_{\Phi} \int_{\Omega} f\left(x, u_{\Phi}\right) v d x \text { for all } v \in W_{0}^{1, p(x)}(\Omega)
$$

where

$$
\lambda_{\Phi}=\frac{\Phi\left(I\left(u_{\Phi}\right)\right)}{\Phi^{\prime}\left(I\left(u_{\Phi}\right)\right) \int_{\Omega} F\left(x, u_{\Phi}\right) d x}>0 .
$$

We have by equation (3.4) that $u_{\Phi}$ is a weak solution of Problem (1.1) and since $u_{\Phi} \neq 0, \lambda_{\Phi}$ is a positive eigenvalue of Problem (1.1).

We affirm that the application $\Phi \mapsto \lambda_{\Phi}$ is well defined. Indeed, let $\Phi_{1}=\Phi_{2}$ then there exists $c>0$ such that $\phi_{1}(r)=c \phi_{2}(r)$, for all $r \geq 0$ where $\phi_{1} \in \Phi_{1}$ and $\phi_{2} \in \Phi_{2}$. So we have $J_{\phi_{1}}=c J_{\phi_{2}}$ and consequently $J_{\Phi_{1}}=c J_{\Phi_{2}}$. This signifies that $J_{\Phi_{1}}$ and $J_{\Phi_{2}}$ have the same critical points. So we conclude that $\lambda_{\Phi_{1}}=\lambda_{\Phi_{2}}$.

Since $\Phi$ is taken arbitrary in $\bar{E}$, it yields that Problem (1.1) has infinitely many positive eigenvalues.

Remark 3.4. The invertibility of the application $\Phi \mapsto \lambda_{\Phi}$ is still an open problem. We do not know if all the solutions of Problem (1.1) can be considered as critical points of the functional $J_{\Phi}$. 


\section{Acknowledgments}

This work is partially supported by the grants of PNR code 8/U23/994 Algeria.

\section{References}

[1] N. Benouhiba, On the eigenvalues of weighted $\mathrm{p}(\mathrm{x})$-laplacian on $\mathbb{R}^{N}$, Nonlinear Analysis, 74 (2011), 235-243.

[2] D. E. Edmunds and J. Rákosník, Sobolev embedding with variable exponent, Studia Mathematica, 143, No. 3,(2000), 267-293.

[3] X. L. Fan, Q. H. Zhang, and D. Zhao, Eigenvalues of p(x)-Laplacian Dirichlet problem, J. Math. Anal. Appl., 302 (2005), 306-317.

[4] O. Kovácik and J. Rákosník, On spaces $\mathrm{Lp}(\mathrm{x})$ and W1;p(x), Czechoslovak Math. J., 41 (1991), 592-618.

[5] M. Mihăilescu and V. Rădulescu, On a nonhomogeneous quasilinear eigenvalue problem in Sobolev spaces with variable exponent, Proc. Amer. Math. Soc., 135 (2007), 2929-2937.

[6] J. Musielak, Orlicz spaces and modular spaces, Lecture notes in Mathematics, 1034, Springer-Verlag, Berlin (1983).

[7] W. Rother, Generelized Emde-Fowler equations of subcritical growth, J. Austral. Math. Soc., 54, Series A (1993),254-262.

[8] M. Růžička, Electrorheological Fluids: Modeling and Mathematical Theory, Springer-Verlag, Berlin (2002). 
